Psychological Medicine, 1988, 18, 275-277

Printed in Great Britain

EDITORIAL

\title{
Defining hysterical symptoms ${ }^{1}$
}

The conventional view would appear to define hysterical symptoms (whether so-called conversion symptoms or hysterical dissociation) in terms of a number of features. The first is the occurrence of a symptom which, if taken at face value, would be an indication of some underlying pathological process; yet no pathology of a conventional kind can be elicited, and the presence of such pathology (whether physical or in the form of a formal psychiatric disorder) seems highly unlikely. An example of something meeting this criterion is a sensory impairment that does not match any recognized anatomical distribution and/or, where evidence can be obtained that the sensory system must be functioning very much better than the patient's claimed symptomatology would suggest (as in the cases reported by Grosz \& Zimmerman, 1965; Miller, 1986). This seems to be the central and essential criterion and it is widely accepted that patients meeting this criterion are encountered from time to time.

There are three other common criteria and all raise problems. It is usually assumed that there must be some secondary gain from the symptom. The difficulty here is that all disorders or illnesses can give rise to some secondary gain (e.g. people feel under an obligation to be especially considerate to those who are ill). Secondary gain therefore raises problems as one of the defining criteria of hysteria since it does not readily distinguish hysteria from true illnesses. A second, classical feature is the alleged presence of 'la belle indifférence' although again this must be regarded as a rather unreliable sign. What evidence there is about anxiety in hysterical patients tends to indicate that levels of general anxiety are, if anything, raised in those with so-called 'conversion hysteria' (Lader \& Sartorius, 1968).

Many authorities on hysteria would probably accept that secondary gain and 'la belle indifférence' are unreliable as signs of hysteria. The final criterion is more central to most views of hysteria. It is that the patient is regarded as experiencing the symptom as 'real' and is not consciously aware that the symptom is being produced and maintained by means other than those of conventional pathological mechanisms. Thus, the hysterical patient with paralysis really is unable to move his paralyzed limbs. It is this latter criterion which distinguishes the hysteric from the malingerer who consciously simulates symptoms. (There can also be a problem in distinguishing hysterical symptoms from hypochondriacal complaints but this is not an issue of such great relevance to present concerns.)

This discussion is principally concerned with the last of the above criteria for hysterical symptoms; that of the subjective reality of the symptom from the point of view of the patient. The: use of this criterion raises a number of difficulties. It entails a judgment about whether or not the patient is aware of the status of the symptom. This is necessarily an unreliable and unsatisfactory judgment because no examiner has access to the content of any patient's consciousness. To maintain this criterion also biases explanations of hysteria towards those that involve unconscious mental mechanisms (as in the psychoanalytic tradition) or which, following Janet (1907), use the concept of dissociation. A model of the mind has to be postulated which involves some mental structure operating outside the realms of normal consciousness which can be used to create the symptom (or the processes underlying it). Not only are such structural models of the mind open to dispute on philosophical grounds (e.g. Ryle, 1949; White, 1967), but they are not amenable to empirical test in any critical way. Of course, verifiable predictions can be derived from such models but not of a kind that would challenge the fundamental basis of the model itself.

The use of the criterion of subjective reality of the symptom from the patient's point of view would be easier to defend if it were possible to show a clear distinction between the behaviour of those with hysterical symptoms and those simulating such symptoms. There is now some evidence on this point. Grosz \& Zimmerman (1965) required a patient with hysterical blindness to view an

' Address for correspondence. Dr Edgar Miller, Department of Clinical Psychology, Addenbrooke's Hospital, Cambridge CB2 2 QQ. 
array of three designs and to select the one that was different in shape from the other two. Blocks of 60 trials were used and it would be expected that, with the position of the key stimulus being varied at random from trial to trial, a blind subject should get about a third of the trials correct by chance. In fact, the subject consistently got very significantly less than a third right, and this remained so even when other non-visual cues were given that might well enable a truly blind subject to predict the position of the correct stimulus on each trial. The only time that their subject scored at chance levels was after the rationale of the experiment had been made aware to him. Similar results using forced choice testing have been reported by others (e.g. Miller, 1968 \& 1986; Pankrantz et al. 1975; Theodor \& Mandelcorn, 1973). Aplin \& Kane (1985) also found that subjects asked to simulate hearing loss gave results on audiometric testing very similar to those reported for hysterical hearing loss.

In a rather different type of experiment Spanos et al. (1985) required subjects to play the role of the prime suspect in a murder enquiry. They were to consider themselves as having been arrested and subjected to interrogation under hypnosis. The experiment was based on the well-known American 'Hillside Strangler' case where a murderer during a psychiatric interview under hypnosis produced responses indicating that he was a multiple personality and later evidence suggested that he was almost certainly faking. When the questions under hypnosis were posed in a particular kind of way, stressing the examiner's wish to get at material not normally consciously accessible in the same way as that used by the psychiatrist in the 'Hillside Strangler' case, a majority of subjects coped with the situation in the same way as the 'Hillside Strangler' by developing a second personality just like hysterical patients with alleged multiple personalities.

Another prediction can be made in relation to multiple personalities. It is well established that learning one set of material (e.g. paired associates) may interfere with the learning and retention of another set of material, especially if the two sets are rather similar. If subjects with multiple personalities really had two separate personalities it might be expected that they would show less interference in this kind of experimental paradigm if the two sets of material were learned while having two separate personalities, than in the case of controls who learned the same material as their one and only personality. Such an experiment has been described by Silberman et al. (1985) who found no such differential effect in the main dependent variables under study. (It may be noted that the authors did in fact claim some minor qualitative differences in performance between the two groups, but this was only on a post hoc basis. Even so, these differences were not statistically significant and there is therefore real doubt that they would re-emerge if the experiment were to be repeated.)

These experimental findings may be considered of interest, in that they indicate that it is extremely difficult to find any way of discriminating between the behaviour of those with hysterical symptoms and the behaviour (actual or anticipated) of those who might be deliberately dissimulating. In the case of the Silberman et al. (1985) experiment, there is also no real difference shown in the behaviour of hysterical subjects as compared with normal controls in the way that might be expected if multiple personality was a true phenomenon. Nevertheless, these experiments cannot finally prove anything about the underlying conscious awareness of hysterical patients. Those with hysterical symptoms could still behave like those deliberately simulating in every possible way, yet the same behaviour could still represent a common endpoint resulting from two entirely different internal processes. While it is impossible to prove finally that any patient with a hysterical symptom is faking, it is equally impossible to prove that he is not, and the available evidence is consistent with the notion that hysterical symptoms might be nothing more than dissimulation. For those who wish to develop an empirically verifiable explanation of hysteria, basing the definition of the phenomenon itself on a criterion that cannot be reliably shown to be present or absent is, at best, highly unsatisfactory. The suggestion being made here is that this criterion be dropped, and that those concerned with hysteria choose to remain agnostic on this point.

One major objection to this conclusion is that there is then no real separation between hysteria and malingering. This could present difficulties in management. In the first place, it can be countered that, if the above arguments are correct, there may not be a clear distinction between hysteria and 
malingering anyway. Distinctions cannot be maintained merely because it would avcid awkward decisions if things worked out that way. Secondly, cases which may be hysterical and/or malingering can be managed using the same general principles. The crucial thing is to ask why the patient is behaving in this way, and then to base management on the answer to this question regardless of whether the symptoms are assumed to have been consciously produced, or not. In a few cases this might involve rejecting them, but for many the sensible response would be to look at what might be done to relieve or modify the precipitating factors.

A consequence of remaining agnostic with regard to the subjective reality of the symptom, is that it then opens the way to an emphasis on models of hysteria which do not have to be based on some form of unconscious mental mechanism, and which can lead much more directly to methods of management. One such general model is that viewing hysterical symptoms as a manifestation of a disturbance in illness behaviour, or an inappropriate adoption of the sick role (e.g. Pilowsky, 1969; Kendell, 1983). Such models do have their problems in that, unless carefully formulated, they can be merely tautologous (e.g. describing a man with hysterical paraplegia who spends his days in a wheelchair as inappropriately adopting the sick-role, could be merely describing the phenomena in different terms). Nevertheless, interesting questions for research can be raised on the basis of this model (Miller, 1987). For example, whether those with hysterical symptoms have had greater opportunity than others to experience the benefits of the sick role (either for themselves or by seeing the consequences of illness in others), or whether they tend to make different attributions in relation to minor physical symptoms? The answers to these questions could then lead on to therapeutic strategies. For example, cognitive therapies as used in depression (e.g. Williams, 1984) might be of value if hysterical subjects tend to make unusual attributions about minor symptoms.

The point here is not to argue that the sick-role/illness behaviour model of hysteria can necessarily be turned into an adequate explanation of the development or maintenance of hysterical symptoms, or even a reasonable approximation of such an explanation. The key issue is that any criteria for the existence of hysterical symptoms are most unsatisfactory if they assume anything about the patients' level of conscious awareness about the true nature of the symptom. Models of hysteria can be developed which do not rely on any such assumption. These can lead to potentially fruitful and testable hypotheses which may advance the understanding and management of hysterical symptoms.

EDGAR MILLER

\section{REFERENCES}

Aplın, D. Y. \& Kane, J. M. (1985). Varables affecting pure tone and speech audiometry in experimentally simulated hearing loss. British Journal of Audiology 19, 219-228.

Grosz, H. J. \& Zimmerman, J. (1965). Experimental analysis of hysterical blindness. Archives of General Psychiatry 13, 255-260.

Janet, P. (1907). The Major Symptoms of Hysteria. Macmillan: New York.

Kendell, R. E. (1983). Hysteria. In Handbook of Psychiatry, Vol. 4., The Neuroses and Personality Disorders (ed. G. F. M. Russell and L. A. Hersov), pp. 232-246. Cambridge University Press: Cambridge.

Lader. M \& Sartorius, N. (1968). Anxiety in patients with hysterical conversion symptoms. Journal of Neurology Neurosurgery and Psychiatry 31, 490-495.

Miller, E. (1968). A note on the memory performance of a patient with unilateral functsonal blindness. Behaviour Research and Therapy 6, 115-116.

Miller, E. (1986). Detectung hysterical sensory symptoms : an elaboration of the forced choice technique. British Journal of Clinical Psychology 25, 231-232.

Miller, E. (1987). Hysteria: its nature and explanation. British Journal of Clinical Psychology (in the press).
Pankrantz, L., Fausti, S. A \& Peed, S. (1975) A forced choice technique to evaluate deafness in the hysterical or malıngering patient Journal of Consultming and Clinical Psychology 43, 421422.

Pilowsky, I. (1969). Abnormal illness behaviour. British Journal of Medical Psychology 42, 347-351.

Ryle, G. (1949). The Concept of Mind. Hutchinson: London.

Silberman, E. K., Weingartner, H., Braun, B. G. \& Post, R. M. (1985). Dissociative states in multiple personality disorder: a quantitative study. Psychiatry Research 15, 25:3-260.

Spanos, N. P., Weekes, J. R. \& Bertrand, L. D. (1985). Multıple personality ' a social psychological perspective. Journal of Abnormal Psychology 94, 362-376.

Theodor, L. H. \& Mandelcorn, M. S. (1973). Hysterical blindness: a case report of a study using a modern psychophysiological technique. Journal of Abnormal Psychology 82, 522-553.

White, A. R. (1967). The Philosophy of Mind. Tandom House: New York.

Williams, J. M. G. (1984). The Psychological Treatment of Depression. Croom Helm: London. 